\title{
ANÁLISE CARDÍACA DE RATOS SUBMETIDOS A EXPOSIÇÃO CRÔNICA DO HERBICIDA ÁCIDO DICLOROFENOXIACÉTICO (2,4-D)
}

Dyovana Gomes Pinheiro, Geovana Letícia Fernandes de Oliveira, Stéfani Nobrega Perrud, Laís de Oliveira Santos Pereira, Luana Victoria Damacena de Carvalho, Millena Aparecida de Oliveira, Rebecca Matiuso Moço, Raissa de Oliveira Mantovani, Gisele Alborghetti Nai, Francis Lopes Pacagnelli

Universidade do Oeste Paulista - UNOESTE, Curso de Fisioterapia, Presidente Prudente/SP. E-mail: dyovana131@hotmail.com

\section{RESUMO}

Um dos herbicidas mais utilizados nas colheitas de cana-de-açúcar do Brasil é o ácido diclorofenoxiacético (2,4-D). Sabe-se que sua ingestão pode causar danos cardiovasculares. O objetivo desse estudo foi avaliar o uso oral do 2,4-D no coração de ratos submetidos à exposição crônica, por meio de parâmetros anatômicos e dosagem da creatina quinase fração músculo-cérebro (CKMB). Trinta ratos Wistar albinos machos, divididos em 3 grupos: GCO ( $n=10)$, GBCO $(n=10)$, e GACO ( $n=10)$. A ração foi exposta $15 \mathrm{~min} /$ dia ao 2,4-D e ofertada durante 6 meses aos animais. Após, foram eutanasiados, os corações dissecados e pesados: átrios, ventrículo esquerdo e direito e o comprimento da tíbia utilizado como normalizador. O sangue foi coletado por punção cardíaca para dosagem da CKMB. Não houve hipertrofia atrial, ventricular direita e esquerda, nem aumento da CKMB no sangue. Concluímos que o 2,4-D não promoveu alterações estruturais anatômicas, nem lesão cardíaca nas concentrações avaliadas.

Palavras-chave: herbicidas, insuficiência cardíaca, cardiotoxicidade, remodelação ventricular.

\section{CARDIAC ANALYSIS OF RATS SUBMITTED TO CHRONIC EXPOSURE OF THE HERBICIDE DICHLOROPHENOXYACETIC ACID (2,4-D)}

\begin{abstract}
One of the most commonly used herbicides in Brazil during the sugar cane harvest is dichlorophenoxyacetic acid $(2,4-D)$. It is known that ingestion of this herbicide can cause cardiovascular damage. The objective of this study was to evaluate the oral use of 2,4-D on the heart of rats submitted to chronic exposure, using anatomical parameters and creatine kinase muscle-brain fraction (CKMB) dosage. Thirty male Wistar albino rats were divided into 3 groups: GCO $(n=10), \operatorname{GBCO}(n=10)$, and GACO $(n=10)$. The animal feed was exposed to 2,4-D for $15 \mathrm{~min} /$ day and offered to the animals for 6 months. After this period, the animals were euthanized, the hearts dissected and weighed: atrium, left and right ventricle, the length of the tibia was used as the normalizer. Blood was collected through cardiac puncture for CKMB dosage. The results did not indicate atrial hypertrophy, right ventricular or left ventricular, or an increase in CKMB in the blood. We conclude that 2,4-D did not promote anatomical structural alterations or cardiac injury at the concentration evaluated.
\end{abstract}

Keywords: herbicides, heart failure, cardiotoxicity, ventricular remodeling.

\section{INTRODUÇÃO}

O Brasil é o maior consumidor de agrotóxicos no mundo ${ }^{1}$. Dentre os meios de exposição existem três vias: dérmica, inalatória e oral ${ }^{2}$. 0 ácido diclorofenoxiacético (2,4-D) é um herbicida de Classe I (Faixa vermelha- mais tóxica), utilizado em plantações de cereais, como o trigo e no
Brasil principalmente em culturas de cana-deaçúcar, para o controle de plantas daninhas, sendo tóxico ao ser humano de forma aguda ou crônica ${ }^{3-5}$.

Dentre os efeitos que os agrotóxicos provocam já está relatado algumas alterações cardiovasculares ${ }^{6}$. Há redução da pressão arterial 
e frequência cardíaca, depressão isquêmica, arritmias, evidenciados ao eletrocardiograma ${ }^{7-8}$. Há relatos de efeitos imunotoxicológicos com o uso de vários tipos de agrotóxicos, dentre ele o diazinon, paraquat, glifosato, glufosinato e atrazina, fosfina, dimetoato que provocaram hemorragias, infiltração de linfócitos, aumento de citocinas, apresentando excesso de radicais livres e de danos oxidativos proteicos incluindo a perioxidação lipídica que ocasiona perda de tecido contrátil, hipertrofia das células miocárdicas e fibrose reparadora ${ }^{9-12}$. Sendo que entre os agrotóxicos que tem maior quantidade de estudos se destaca o glifosato, porém não há relatos na literatura sobre estes aspectos prejudiciais do 2,4-D, tornando importante esse estudo para melhor conhecimento sobre 0 mesmo.

O objetivo desse estudo é avaliar o uso oral de baixa e alta concentração do herbicida 2,4-D na remodelação cardíaca em ratos submetidos à exposição crônica.

\section{METODOLOGIA}

\section{Protocolo experimental}

Este estudo foi aprovado pelo comitê de ética da Unoeste, Comitê de Ética de Uso de Animais (CEUA) sob o protocolo número 4259. E também se apresenta em conformidade com os princípios de cuidados com animais de laboratório formulado pelo Colégio Brasileiro de Experimentação Animal (COBEA).

Para a realização dos experimentos, foram utilizados 30 ratos Wistar albinos, machos, adultos, com peso entre $150-200 \mathrm{~g}$, fornecidos pelo Biotério Central da UNOESTE, esses ratos foram alocados em gaiolas plásticas individuais, medindo $30 \times 16 \times 19$ centímetros, a temperatura média de $22 \pm 2^{\circ} \mathrm{C}$, com ciclos de $12 \mathrm{~h}$ de luminosidade, sendo das $7 \mathrm{~h} 00$ as $19 \mathrm{~h} 00$ (período claro) e 19 h00 as 7 h00 (período escuro).

O herbicida utilizado foi 0 ácido diclorofenoxiacético foi com 2,4-D (Nortox S.A., Arapongas, PR, Brasil), registrado no Ministério da Agricultura, Pecuária e Abastecimento - MAPA - sob o no 03009, com a seguinte composição: sal de dimetilamina de (2,4-dichlorophenoxy) acetic acid (2,4D): $806 \mathrm{~g} / \mathrm{L}(80,6 \% \mathrm{~m} / \mathrm{v})$, equivalente acid de 2,4D: $670 \mathrm{~g} / \mathrm{L}(67,0 \% \mathrm{~m} / \mathrm{v})$ e ingredientes inertes: $424 \mathrm{~g} / \mathrm{L}(42,4 \% \mathrm{~m} / \mathrm{v})$.

Os animais foram divididos aleatoriamente em três grupos: GCO - grupo controle oral $(n=10)$ : receberam ração nebulizada com água destilada por aproximadamente 15 minutos, GBCO - grupo de baixa concentração oral $(n=10)$ : receberam ração nebulizada com herbicida com $3,71 \times 10^{-3} \mathrm{~g}$ de ingrediente ativo por hectare (g.i.a/ha) por aproximadamente 15 min e GACO - grupo de alta concentração oral $(n=10)$ : receberam ração nebulizada com herbicida com 9,28 $\times 10^{-3} \mathrm{~g}$ de ingrediente ativo por hectare (g.i.a/ha), por aproximadamente 15 $\min$.

\section{Protocolo de exposição ao herbicida 2,4-D}

A manipulação do herbicida 2,4-D foi realizada mediante a utilização dos seguintes equipamentos de proteção individual (EPI): luvas de borracha, óculos de proteção e máscaras com filtro para gases ${ }^{13}$.

O protocolo de experimentação contou com duas caixas $(32 \times 24 \times 32 \mathrm{~cm})$ cada uma ligada a um nebulizador ultrassônico da marca Pulmosonic Star ${ }^{\circledR 14}$. O tempo de exposição foi de aproximadamente $15 \mathrm{~min}$ nas segundas-feiras e quintas-feiras, para a ração, tempo este necessário para que toda a solução seja nebulizada e a troca da ração para os animais foi realizada as terças e sextas-feira. A ração dos animais de todos os grupos foi pesada a cada troca para a avaliação da quantidade de ingestão.

Todos animais foram expostos durante 6 meses e após este período foram eutanasiados. A anestesia e eutanásia foram realizadas com Tiopental sódico (Syntec, EUA), nas doses de 40 $\mathrm{mg} / \mathrm{Kg}$ e $100 \mathrm{mg} / \mathrm{Kg}$ de peso, respectivamente, administrado na cavidade peritoneal. Os indicativos de morte foram a ausência de movimentos respiratórios, batimentos cardíacos e perda dos reflexos ${ }^{15}$.

\section{Avaliação da Cardiotoxidade}

Análise dos parâmetros Anatômicos

Os animais foram eutanasiados e seus órgãos (coração) foram retirados e pesados. $O$ peso úmido do ventrículo esquerdo (VE), ventrículo direito (VD) e dos átrios foi normalizado para peso corpóreo final do rato (PC), que foi utilizado como índice de hipertrofia ventricular.

\section{Analise da CKMB}

O sangue foi coletado por punção cardíaca para bioquímica sérica da creatina quinase fração músculo-cérebro (CKMB), foi realizada sem anticoagulantes, em tubos 
(Vacutainer $\left.{ }^{\circledR}\right)$. O sangue foi centrifugado a 3000 rpm ( $g=1257)$ e foram utilizados microtubos plásticos para armazenar o soro obtido, sendo mantido em $-20^{\circ} \mathrm{C}$. Através do método cinético UV automatizado (Cobas C111, Roche ${ }^{\circledR}$ ) foi realizada a bioquímica sérica.

Analise estatística

A normalidade dos dados foi avaliada pelo teste Shapiro-Wilk. Para os dados que forem paramétricos foi utilizado ANOVA seguido de Tukey, para os dados não paramétricos foi utilizado Kruskal-Wallis seguido do pós teste de Dunns. Os dados foram expressos em média \pm desvio padrão (DP), mediana e intervalo interquartil $25-75 \%$. Foi utilizado o software
GraphPad Prism. O nível de significância considerado foi de $p<0,05$.

\section{RESULTADOS}

Durante o experimento, um rato do grupo GACO foi eutanasiado por apresentar otite, portanto as análises foram feitas com os vinte e nove ratos restantes. A Tabela 1 mostra as variáveis anatômicas nos três grupos, e as relações de normalidades com o comprimento da tibia. Em relação à análise dos parâmetros anatômicos não houve diferença estatística na comparação entre os grupos (Tabela 1).

A Tabela 2 mostra a análise da CK-MB nos 3 grupos, e não houve diferença estátistica. (Tabela 2)

Tabela 1. Parâmetros anatômicos.

\begin{tabular}{lcccc|}
\hline VARIAVEIS & $\begin{array}{c}\text { GCO } \\
(\mathrm{n}=10)\end{array}$ & $\begin{array}{c}\text { GBCO } \\
(\mathrm{n}=10)\end{array}$ & $\begin{array}{c}\text { GACO } \\
(\mathrm{n}=9)\end{array}$ & $\boldsymbol{p}$ \\
\hline PCF $(\mathrm{g})$ & $466,7 \pm 26,72$ & $490,1 \pm 16,92$ & $465,7 \pm 26,10$ & 0,05 \\
VE $(\mathrm{g})$ & $1,05 \pm 0,05$ & $1,00 \pm 0,06$ & $1,02 \pm 0,06$ & 0,23 \\
VE/Comp tíbia $(\mathrm{g} / \mathrm{cm})$ & $0,22 \pm 0,017$ & $0,22 \pm 0,01$ & $0,23 \pm 0,015$ & 0,43 \\
VD (g) & $0,22 \pm 0,02$ & $0,23 \pm 0,03$ & $0,23 \pm 0,02$ & 0,59 \\
VD/Comp tibia (g/cm) & $0,04 \pm 0,008$ & $0,05 \pm 0,007$ & $0,05 \pm 0,006$ & 0,69 \\
Átrios (g) & $0,09 \pm 0,017$ & $0,10 \pm 0,03$ & $0,09 \pm 0,02$ & 0,47 \\
Átrios/Comp tibia (g/cm) & $0,01 \pm 0,003$ & $0,02 \pm 0,007$ & $0,02 \pm 0,006$ & 0,33 \\
\hline
\end{tabular}

Dados como média \pm DP. GCO: Grupo controle oral. GBCO: Grupo baixa concentração oral. GACO: Grupo alta concentração oral. PCF: peso corporal final. VE: Ventrículo Esquerdo. VE/comp tíbia: relação ventrículo esquerdo com comprimento da tíbia. VD: Ventriculo Direito. VD/comp tíbia: relação ventrículo direito com comprimento da tíbia. Átrios/compr tíbia: relação dos átrios com o comprimento da tíbia. ANOVA seguido de Tukey ou Kruskal-Wallis seguido de Dunns.

Tabela 2. Dados CKMB expressos em mediana (intervalo interquartil: 25-75\%).

\begin{tabular}{lcccc}
\hline VARIAVEIS & $\begin{array}{c}\text { GCO } \\
(n=10)\end{array}$ & $\begin{array}{c}\text { GBCO } \\
(n=10)\end{array}$ & $\begin{array}{c}\text { GACO } \\
(n=9)\end{array}$ & $p$ \\
\hline CKMB & $526,6(356,2-687,8)$ & $479,3(432,6-833,1)$ & $388,3(317,4-460,0)$ & 0,1942 \\
\hline
\end{tabular}

Dados da mediana (intervalo interquartil). CKMB: Creatina quinase músculo-cérebro. Kruskal-Wallis seguido de Dunns.

\section{DISCUSSÃO}

Neste estudo observamos que não houve hipertrofia das câmaras cardíacas e nem lesão miocárdica, demonstrando que a ingestão do herbicida 2,4-D não influenciou nessas variáveis.
Em outro estudo, o Grupo controle recebeu $1 \mathrm{~mL}$ água destilada via gavagem oral uma vez por dia. Já os três grupos tratados com 2,4-D receberam respectivamente uma dose de nível $15 \mathrm{mg} / \mathrm{Kg}$ para o G1, $75 \mathrm{mg} / \mathrm{Kg}$ para o G2 e 
$150 \mathrm{mg} / \mathrm{Kg}$ para o G3. As doses de 2,4-D foram administradas oralmente através de uma seringa com a ajuda de uma broca esofágica num período de 4 semanas, sendo que as soluções foram administradas no período da manhã. Com isso foi demonstrado que a exposição subcrônica do 2,4D obteve efeitos tóxicos nos rins (aumento do peso do rim, lesões histopatológicas) e no fígado (aumento do peso do fígado, aumento das enzimas hepáticas) ${ }^{17}$. A exposição crônica em ratos é manifestada por diminuição do ganho de peso corporal, alteração do peso dos órgãos e parâmetros hematológicos e outras alterações bioquímicas ${ }^{18}$. Há contatação que o 2,4-D pode induzir efeitos patológicos deletérios nos órgãos vitais, incluindo alterações pré-neoplásicas no fígado de ratos Sprague-Dawley ${ }^{19}$.

Foi demonstrado também que a exposição oral ao 2,4-D em concentrações de 100 e $200 \mathrm{mg} / \mathrm{Kg}$ em dois grupos de ratos machos por 30 dias, provocou diminuição nos níveis séricos de testosterona, alterações histológicas nos testículos, e diminuição de espermatozoides, indicando a toxicidade, mostrando os danos que os herbicidas podem causar em todo organismo ${ }^{20}$. De acordo com Pasandi et al. ${ }^{21}$ após o tratamento de células isoladas com concentrações de 2,4-D de 1, 10 e $10 \mu \mathrm{M}$ para 1,3 e $5 \mathrm{mM}$ por $48 \mathrm{~h}$, houve neurotoxicidade, ocorrendo a redução da proliferação de células perineurais e de Schwann.

Em relação a outros herbicidas quando a composição contem Glufosinato ou um Surfactante, pode ocorrer efeitos cardiovasculares diferentes, de acordo com a dose e o componente, tendo efeitos cardiossupressores ou cardioestimulatórios, o que pode-se considerar um determinante para os efeitos diretos ao coração ${ }^{22}$. Já no estudo de Gress et al. ${ }^{7}$ o herbicida Roundup administrado em ratos com doses altas (50 e 500 ppm de concentração) ocasionou bloqueios de condução e alterações elétricas cardíacas.

Os achados desse estudo diferenciam-se dos demais encontrados na literatura por haver poucos relatos que tragam os efeitos do 2,4-D no coração, tornando relevante o assunto, sendo importante novos estudos que tragam outros parâmetros a serem avaliados para melhor conhecimento.

\section{CONCLUSÃO}

O uso oral do herbicida 2,4-D não promoveu alterações estruturais anatômicas e também não houve lesão cardíaca significativa nas concentrações avaliadas.

\section{CONFLITOS DE INTERESSE}

Os autores declaram não haver qualquer potencial conflito de interesses que possa interferir na imparcialidade deste trabalho científico.

\section{REFERÊNCIAS}

1. Ministério do Meio Ambiente. Segurança Química, agrotóxicos. c2017. Brasil. Acesso em: 8 set 2018]. Disponível em: http://www.mma.gov.br/segurancaquimica/gestao-das-substanciasquimicas/produtos-agrot\%C3\%B3xicos

2. Almeida MD, Cavendish TA, Bueno PC, Ervilha IC, Gregório LS, Kanashiro NBO et al. A flexibilização da legislação brasileira de agrotóxicos e os riscos à saúde humana: análise do Projeto de Lei no 3.200/2015. Cad Saúde Púb. 2017;33(7): e00181016. DOI: https://doi.org/10.1590/0102-311×00181016

3. Machi AR, Ferrari L, Mendes A, Arthur V. Efeitos da radiação gama $(60 \mathrm{Co})$ sobre o herbicida 2,4-D no controle de trapoeraba (Commelina virginica.L). Rev Verde. 2014;9(3):258-262.

4. Baumgartner D, Souza EG, Coelho SRM, Maggi MF. Correlation between 2,4-D herbicide residues and soil attributes in southern of Brazil. Rev Ciênc Agron. 2017;48(3):428-437. DOI: https://doi.org/10.5935/1806-6690.20170050

5. Amarante Junior OP, Santos TCR, Brito NM, Ribeiro ML. Revisão das propriedades, usos e legislação do Ácido 2,4-diclorofenoxiacético (24D). Cad Pesq. 2002;13(1):60-70.

6. Chan YC, Chang SC, Hsuan SL, Chien MS, Lee WC, Kang JJ et al. Cardiovascular effects of herbicides and formulated adjuvants on isolated rat aorta and heart. Toxicol In Vitro. 2007;21(4):595-603. DOI: 
7. Gress S, Lemoine S, Puddu PE, Séralini GE, Rouet R. Cardiotoxic electrophysiological effects of the herbicide Roundup in rat and rabbit ventricular myocardium in vitro. Cardiovasc Toxicol. 2015; 15(4):324-335. DOI: https://doi.org/10.1007/s12012-014-9299-2

8. Abdolghaffari $A H$, Baghaei $A$, Solgi R, Gooshe $M$, Baeeri $M$, Navaei-Nigjeh $M$ et al. Molecular and biochemical evidences on the protective effects of Triiodothyronine against phosphineinduced cardiac and mitochondrial toxicity. Life Sci. 2015;139:30-39. DOI: https://doi.org/10.1016/j.Ifs.2015.07.026

9. Razavi $B M$, Hosseinzadeh $H$, Imenshahidi $M$, Malekian M, Ramezani M, Abnous K. Evaluation of protein ubiquitylation in heart tissue of rats exposed to diazinon (an organophosphate insecticide) and crocin (an active saffron ingredient): role of hif- $1 \alpha$. Drug Res (Stuttg). 2015;65(11):561-566. DOI: https://doi.org/10.1055/s-0034-1384533

10. Dhivya Vadhana MS, Siva Arumugam S, Carloni M, Nasuti C, Gabbianelli R. Early life permethrin treatment leads to long-term cardiotoxicity. Chemosphere. 2013;93(6):10291034.

DOI:

https://doi.org/10.1016/i.chemosphere.2013.05. $\underline{073}$

11. Vedhana MS, Nasuti C, Gabbianelli R. Purine bases oxidation and repair following permethrin insecticide treatment in rat heart cells. Cardiovasc Toxicol. 2010;10(3):199-207. DOI: https://doi.org/10.1007/s12012-010-9079-6

12. Amara IB, Soundani N, Hakim A, Troudi A, Zeghal KM, Boudawara $T$, et. al. Proctetive effects of vitamin $\mathrm{E}$ and selenium against dimethoateinduced cardiotoxicity in vivo: biochemical and histological studies. Environ Toxicol, 2013;28(11):630-43. DOI: https://doi.org/10.1002/tox.20759

13. Carvalho NC. Diretrizes para Aplicação de Herbicida na Suzano Papel e Celulose. 2011. Acesso em: 18 jun 2018. Disponível em: http://www.dialogoflorestal.org.br/download.ph p?codigoArquivo $=233$
14. Domingues RR, Mesquita GL, Cantarella H, Junio DM. Suscetibilidade do capim-colonião e de cultivares de milho ao flúor. Bragantia. 2011;70(4):729-36. DOI: https://doi.org/10.1590/S0006$\underline{87052011000400001}$

15. Ministério da saúde. Fundação Oswaldo Cruz - FIOCRUZ. Curso de Manipulação de Animais de Laboratório. Centro de Pesquisas Gonçalo Muniz. 2005. Acesso em: 25 mar 2005. Disponível em: http://www.bioteriocentral.ufc.br/arquivos/apos tilha manipulacao.pdf.

16. Adriana J, Cicogna AC, Engel LE, Alda MA, Tomasi LC, Giuffrida R et al. Efeitos do hormônio do crescimento na remodelação cardíaca durante treinamento resistido em ratos. Arq Bras Cardiol. 2016;106(1):18-25. DOI: https://doi.org/10.5935/abc.20160003

17. Tayeb W, Nakbi A, Trabelsi M, Miled A, Hammami M. Biochemical and histological evaluation of kidney damage after sub-acute exposure to 2,4-dichlorophenoxyacetic herbicide in rats: involvement of oxidative stress. Toxicol Mechan Meth. 2012;22(9):696-704. DOI: https://doi.org/10.3109/15376516.2012.717650

18. Aydin H. Effects of 2,4-Dichlorophenoxyacetic Acid(2,4-D) on serum glucose level and kidney function activities in subchronically exposed rats. Fresenius Environm Bull. 2006;15(2):105-109.

19. Hassanein KMA. Histopathological effects of 2,4-dichlorophenoxyacetic acid on SpragueDawley rats with special reference to its possible carcinogenicity. Veterinary World. 2012;5(1):2430. DOI: https://doi.org/10.5455/vetworld.2012.24-30

20. Marouani N, Tebourbi O, Cherif D, Hallegue $D$, Yacoubi MT, Sakly $M$ et al. Effects of oral administration of 2,4-dichlorophenoxyacetic acid (2,4-D) on reproductive parameters in male Wistar rats. Environ Sci Pollut Res Int. 2017;24(1):519-526. DOI: https://doi.org/10.1007/s11356-016-7656-3

21. Pasandi MS, Shirazi FH, Gholami MR, Salehi $H$, NNajafzadeh N, Mazani M, Hamidabadi HG, Niapour N. Epi/perineural and Schwann Cells as Well as Perineural Sheath Integrity are Affected 
Following 2,4-D Exposure. Neurotox Res. 2017;32(4):624-638. DOI: https://doi.org/10.1007/s12640-017-9777-y

22. Koyama K, Koyama K, Goto K. Cardiovascular effects of a herbicide containing glufosinate and a surfactant: in vitro and in vivo analyses in rats. Toxicol Appl Pharmacol. 1997;145(2):409-414. DOI: https://doi.org/10.1006/taap.1997.8196
Recebido para publicação em 09/08/2018 Revisado em 21/08/2018

Aceito em 17/09/2018 